\title{
The high energy X-ray tail of $\eta$ Car revealed by BeppoSAX ${ }^{\star}$
}

\author{
R. F. Viotti ${ }^{1}$, L. A. Antonelli ${ }^{2}$, C. Rossi ${ }^{3}$, and S. Rebecchi ${ }^{4}$ \\ ${ }^{1}$ Istituto di Astrofisica Spaziale e Fisica Cosmica, CNR, Area di Ricerca Tor Vergata, Via del Fosso del Cavaliere 100, \\ 00133 Roma, Italy \\ 2 INAF-Osservatorio Astronomico di Roma, Via di Frascati 33, 00040 Monte Porzio Catone, Roma, Italy \\ ${ }_{3}$ Dipartimento di Fisica, Università La Sapienza, Piazzale Aldo Moro 3, 00185 Roma, Italy \\ 4 ASI Science Data Center (ASDC), c/o ESA-ESRIN, Via Galileo Galilei, 00044 Frascati, Roma, Italy
}

Received 27 December 2003 / Accepted 10 February 2004

\begin{abstract}
We report on the June 2000 long (100 ks) BeppoSAX exposure that unveiled a new very high energy component of the X-ray spectrum of $\eta$ Car above $10 \mathrm{keV}$ extending to at least $50 \mathrm{keV}$. We find that the $2-150 \mathrm{keV}$ spectrum is best reproduced by a thermal + non-thermal model. The thermal component dominates the $2-10 \mathrm{keV}$ spectral range with $k T_{h}=5.5 \pm 0.3 \mathrm{keV}$ and $\log \mathrm{NH}_{h}=22.68 \pm 0.01$. The spectrum displays a prominent iron emission line centred at $6.70 \mathrm{keV}$. Its equivalent width of $0.94 \mathrm{keV}$, if produced by the thermal source, gives a slightly sub-solar iron abundance $([\mathrm{Fe} / \mathrm{H}]=-0.15 \pm 0.02)$. The high energy tail above $10 \mathrm{keV}$ is best fitted by a power law with a photon index of $2.42 \pm 0.04$. The integrated 13-150 keV luminosity of $\sim 12 L_{\odot}$ is comparable to that of the $2-10 \mathrm{keV}$ thermal component $\left(19 L_{\odot}\right)$. The present result can be explained, in the $\eta$ Car binary star scenario, by Comptonisation of low frequency radiation by high energy electrons, probably generated in the colliding wind shock front, or in instabilities in the wind of the S Dor primary star. It is possible that the high energy tail had largely weakened near the minimum of the 5.53 yr cycle. Probably, the thermal component has a longer recovery time like that of the highest excitation optical emission lines. Both features can be associated with the large absorption measured by BeppoSAX at phase 0.05 .
\end{abstract}

Key words. radiation mechanisms: non-thermal - stars: individual: $\eta \mathrm{Car}$ - stars: winds, outflows - X-rays: stars

\section{Introduction}

The peculiar southern object $\eta$ Car is one of the most remarkable variables in our Galaxy due to the dramatic changes in its brightness. In 1843 it was the second brightest star in the sky, then decreased to the eighth magnitude by the end of the 19th century (e.g. Viotti 1995). During the last century $\eta$ Car slowly and irregularly re-brightened to the present $V \simeq$ 5. According to the current distance estimates, $\eta$ Car has a bolometric magnitude around $5 \times 10^{6} L_{\odot}\left(2 \times 10^{40} \mathrm{erg} \mathrm{s}^{-1}\right.$, e.g. Hillier et al. 2002). A mass loss rate of $10^{-3 /-4} M_{\odot} \mathrm{yr}^{-1}$ or larger has been estimated from observations (e.g. Hillier et al. 2002; van Boekel et al. 2003; Pittard \& Corcoran 2002; Andriesse et al. 1978).

Optical spectroscopic observations unveiled a peculiar cyclic behaviour, showing regularly repeating excitation minima with a period of 5.53 years (Damineli et al. 2000). A similar behaviour was also found at other wavelength bands, from radio to X-rays, which is commonly interpreted in terms of a

Send offprint requests to: $\mathrm{R}$. Viotti, e-mail: uvspace@rm.iasf.cnr.it

* Based on space observations collected with the BeppoSAX X-Ray Astronomy Satellite which is a program of the Agenzia Spaziale Italiana with participation of the Netherlands Agency for Aerospace Programs. highly eccentric binary model composed of an S Dor-type very luminous primary star, and an unseen early type secondary star. The binary system interacts through colliding winds producing the observed high temperature luminous X-ray emission (e.g. Ishibashi et al. 1999; Corcoran et al. 2001).

Recently, thanks to the BeppoSAX unique broad-band $\mathrm{X}$-ray coverage we were able to detect, for the first time, $\eta \mathrm{Car}$ above $10 \mathrm{keV}$ (Viotti et al. 1998, 2002a, Paper I). We reported the December 1996 observation showing a $13-20 \mathrm{keV}$ flux in excess of the extrapolated $5 \mathrm{keV}$ thermal spectrum that dominates the $2-10 \mathrm{keV}$ range. The presence of a high energy tail was confirmed by the following BeppoSAX observations of 31 December 1999-2 January 2000 (Rebecchi et al. 2001). In particular, in June 2000 a 100 ks exposure showed that the tail was probably non-thermal and extending to at least $50 \mathrm{keV}$ (Viotti et al. 2002b). In this work we analyse in detail the latter observation in order to investigate the origin of these very high energy photons, and compare with the previous BeppoSAX observations of $\eta$ Car. The results are summarised in Table 1.

\section{Observations}

The BeppoSAX satellite observed $\eta$ Car on June 21-23, 2000 with its Narrow Field Instruments (NFI). NFI include a Low Energy Concentrator Spectrometer (LECS) 
Table 1. Summary of the BeppoSAX observations during 1996-2000. Phase one corresponds to the spectroscopic minimum of 1998.0.

\begin{tabular}{cccccccc}
\hline \hline Date & $\begin{array}{c}\text { JD } \\
2400000+\end{array}$ & Phase & $\begin{array}{c}\text { PDS (13-20 keV) } \\
\mathrm{s}^{-1}\end{array}$ & $\begin{array}{c}\text { Weq(Fe-k) } \\
\mathrm{keV}\end{array}$ & $\begin{array}{c}f_{\mathrm{X}}^{0}(2-10 \mathrm{keV}) \\
\mathrm{erg} \mathrm{cm}^{-2} \mathrm{~s}^{-1}\end{array}$ & $\begin{array}{c}k T_{h} \\
\mathrm{keV}\end{array}$ & $\begin{array}{c}\mathrm{NH}_{h} \\
\mathrm{~cm}^{-2}\end{array}$ \\
\hline 1997.00 & 50447 & 0.83 & $0.15 \pm .05$ & $1.05 \pm 0.06$ & $9.4 \times 10^{-11}$ & $4.8 \pm 0.1$ & $4.3 \pm 0.1$ \\
1998.21 & 50891 & 1.05 & $-0.04 \pm .05$ & $1.41 \pm 0.09$ & $12.7 \times 10^{-11}$ & $4.4 \pm 0.2$ & $15.4 \pm 0.4$ \\
2000.00 & 51545 & 1.37 & $0.16 \pm .04$ & $1.01 \pm 0.06$ & $8.4 \times 10^{-11}$ & $5.1 \pm 0.2$ & $3.8 \pm 0.1$ \\
2000.48 & 51718 & 1.46 & $0.17 \pm .03$ & $0.96 \pm 0.04$ & $9.3 \times 10^{-11}$ & $5.5 \pm 0.3$ & $4.9 \pm 0.1$ \\
\hline
\end{tabular}

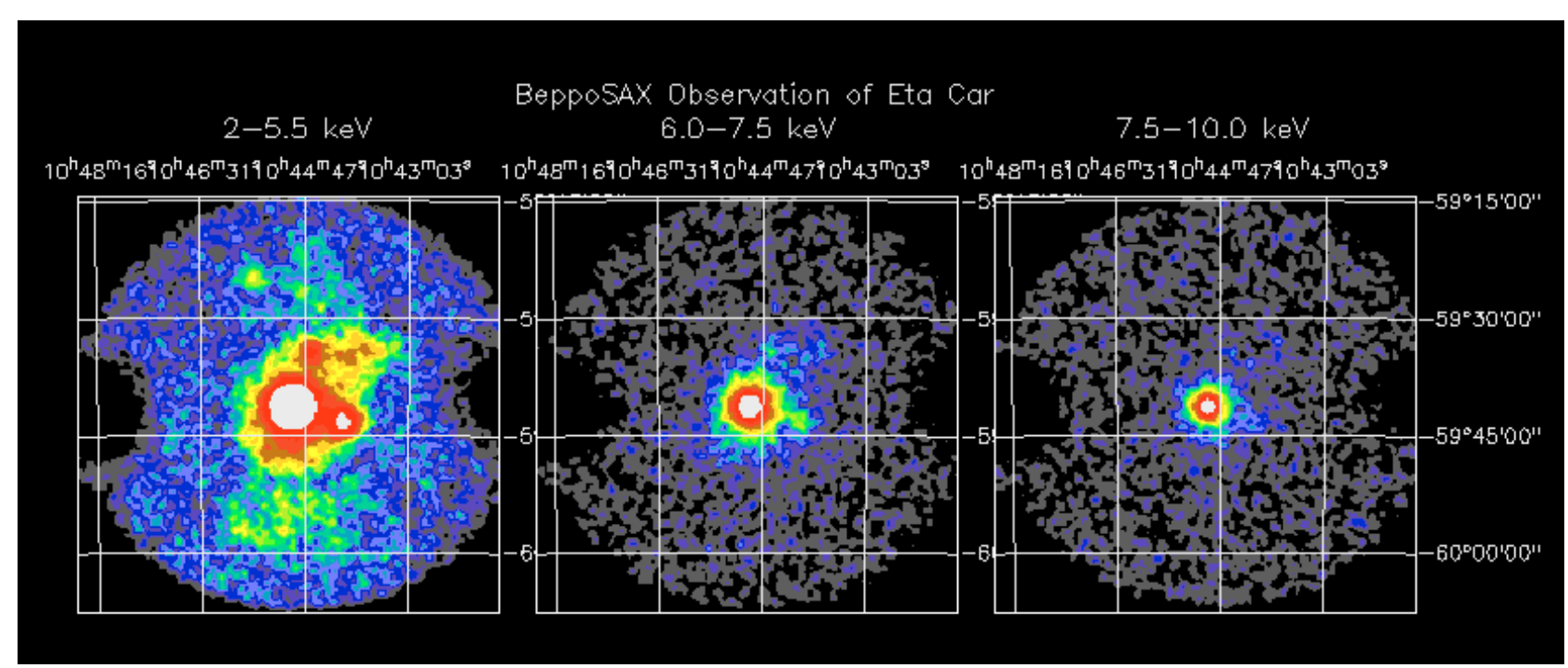

Fig. 1. The BeppoSAX MECS images of the $\eta$ Car region on 2000 June 21-23, in three energy bands: (from left to right) $2-5.5 \mathrm{keV}$ (softer), 6-7.5 keV (iron line), and 7.5-10 keV (harder). North is up and east to the left; the size of the image field is 54 arcmin. The second brightest source to the west of $\eta$ Car is the W-R star HD 93162 (WR25).

(Parmar et al. 1997), and three Medium Energy Concentrator Spectrometers (MECS) (Boella et al. 1997) at the foci of four X-ray telescopes, a Phoswich Detector System (PDS) (Frontera et al. 1997) and a High Pressure Gas Scintillation Proportional Counter (HPGSPC) (Manzo et al. 1997). LECS and MECS units have imaging capabilities and cover the $0.1-10 \mathrm{keV}$ and $1.5-10 \mathrm{keV}$ energy ranges, respectively. PDS covers the 12-200 keV band, while HPGSP covers the 7-60 keV energy range. They are both collimated instruments with a field of view of 1.3 and 1.1 degrees, respectively. The PDS collimators are rocked back and forth by about $3^{\circ}$ to allow the simultaneous monitoring of the source and background. During 2000 HPGSP was switched off. $\eta$ Car has been observed for a nominal exposure time of $100 \mathrm{ks}$. The effective exposures were $80.8 \mathrm{ks}$ for MECS Nos. 2 and 3 (MECS No. 1 was not in operation), and $33.4 \mathrm{ks}$ for PDS. The corresponding count rates were, MECS: $0.639 \pm 0.003$, and PDS: $0.165 \pm 0.032 \mathrm{~s}^{-1}$. The LECS image will not be discussed here, as this paper is devoted to the analysis of the compact hard component which dominates beyond $\sim 2 \mathrm{keV}$. The observation date corresponds to phase 1.457 of the spectral variation cycle of $5.53 \mathrm{yr}$ (Damineli et al. 2000), assuming $\Phi=1$ for the 1998.0 minimum.

Figure 1 shows the MECS images of the region around $\eta$ Car using photon events selected from three different energy bands, in order to trace the softer and harder X-ray sources in the field and to map the regions emitting in the $6.7 \mathrm{keV}$ iron line. It is evident in the figure that $\eta$ Car is the hardest and most luminous object in the region. Some other sources in the field present a non negligible residual in the $7.5-10 \mathrm{keV}$ range and at the iron line, the most interesting one being the Wolf-Rayet star HD 93162/WR 25 (WN6+O4), to the West of $\eta$ Car.

This observation, in particular, shows that $\eta$ Car is the main, and, most probably, unique contributor to the flux observed with the PDS instrument. As discussed in Paper I the PDS field of view also includes the X-ray pulsar 1E 10148.1-5937; but, according to Oosterbroek et al. (1998) and Tiengo et al. (2002) its spectrum is softer and the flux above $10 \mathrm{keV}$ is much fainter than that of $\eta$ Car. Also the lack of intense diffuse emission in the high energy MECS band seems not to support the hypothesis of an extended very high energy emission due to the interaction of the winds from the massive stars in the Carina Nebula.

\section{Spectral analysis}

The MECS spectrum of $\eta$ Car was extracted within a circular region centred on the star with a radius of 4 arcmin. To take into account possible contamination from the nebular Xray emission, we extracted the background from a coronal region around $\eta$ Car with a 4.3 arcmin inner radius and 11.3 arcmin outer radius. We also excluded from the selected area two circular areas of 4 arcmin in radius centred on the strong $\mathrm{X}$ ray sources HD 93162 (WN6+O4) and HD 93250 (O3.5V). The background-subtracted spectrum of $\eta$ Car and the local 


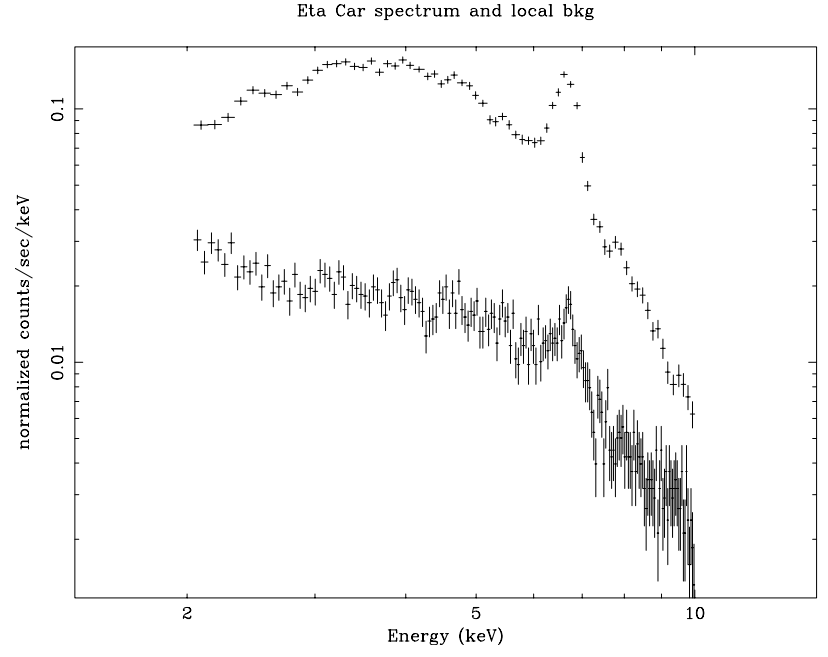

Fig. 2. The BeppoSAX MECS background-subtracted count rates (in $\mathrm{s}^{-1} \mathrm{keV}^{-1}$ ) of $\eta$ Car in June 2000 compared with the local nebular background spectrum.

nebular spectrum normalised to the same area are shown in Fig. 2. The MECS background subtracted spectrum of $\eta$ Car has been rebinned in order to have at least 20 source photons per energy bin.

The background-subtracted PDS data have been rebinned up to $50 \mathrm{keV}$ following the standard procedure. Above $50 \mathrm{keV}$ the data were binned to have a $2 \sigma$ count rate in each bin. As shown in Fig. 3, $\eta$ Car is detected at the $\geq 3 \sigma$ level up to $45 \mathrm{keV}$, and at the $2 \sigma$ level at higher energies. $\eta$ Car had already been detected by PDS in December 1996 and December 1999, but only in the 13-20 keV range due to the shorter exposure times. A PDS upper limit was derived from the March 1998 observation. Table 1 reports the $13-20 \mathrm{keV}$ PDS count rates for the four BeppoSAX observations.

The extracted MECS+PDS spectrum of June 2000 was analysed using the XSPEC spectral fitting software package. We started by analysing MECS alone in the $2-10 \mathrm{keV}$ energy range which is dominated by the hard core component. The MECS spectrum was first fitted with a thermal model (MEKAL) leaving iron abundance free to vary. We obtained the following best fit values of the parameters: $k T_{h}=5.51 \pm$ $0.25 \mathrm{keV}, \mathrm{NH}_{h}=4.82 \pm 0.12 \times 10^{22} \mathrm{~cm}^{-2}$, and a logarithmic iron abundance of $[\mathrm{Fe} / \mathrm{H}]=-0.15 \pm 0.02$. The reduced $\chi^{2}$ was 1.749 (for 64 degrees of freedom), partly due to the small instrumental energy mismatch at $6.7 \mathrm{keV}$ discussed in Paper I. The source temperature is close to that measured in the MECS spectra of December 1996 and March 1998, and reported in Paper I. In June 2000 the $2-10 \mathrm{keV}$ energy distribution is close to that of December 1996, with nearly the same $\mathrm{NH}_{h}$. As discussed in Paper I, $\mathrm{NH}_{h}$ was much larger in March 1998 during $\eta$ Car's egress from the X-ray minimum event $(\Phi=1.05$; see also Fig. 6 below $)$.

As described in Paper I, we have also tried to model the observed MECS spectrum using an absorbed bremsstrahlung model and a Gaussian line. We obtained for the iron line a peak energy of $6.743 \pm 0.001 \mathrm{keV}$ and an equivalent width of $0.964 \mathrm{keV}$, with values for $k T_{h}(5.70 \pm 0.14)$ and

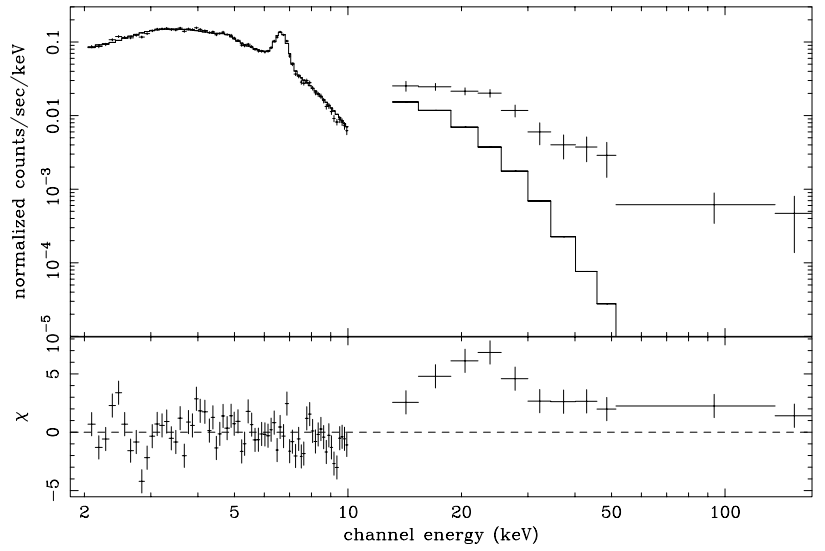

Fig. 3. Upper panel: results of the fit of the June 2000 BeppoSAX MECS background-subtracted spectrum with a one-temperature $(5.51 \mathrm{keV})$ MEKAL model. The spectral model is extrapolated to the PDS energy range to show the high energy flux excess. The residuals are plotted in the lower panel.

$\mathrm{NH}_{h}\left((4.91 \pm 0.10) \times 10^{22} \mathrm{~cm}^{-2}\right)$ similar to those derived from the previous MEKAL model. As reported by Viotti et al. (2002b), The equivalent width of the iron line is consistent with that of December 1996 (1.05 keV) and of December 1999 (1.01 keV), but smaller than that measured in March 1998 (see Table 1). The peak energy of the line, corrected for the $+0.04 \mathrm{keV}$ miscalibration discussed in Paper I, suggests that the line is mostly emitted by the hot plasma, without any important contribution from the $6.4 \mathrm{keV}$ fluorescent line. The reduced $\chi^{2}$ was 1.96 in the range $2-10 \mathrm{keV}$. The integrated absorbed and unabsorbed fluxes in the $2-10 \mathrm{keV}$ en-

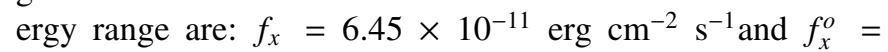
$9.34 \times 10^{-11} \mathrm{erg} \mathrm{cm}^{-2} \mathrm{~s}^{-1}$, respectively. The latter corresponds to $19 L_{\odot}$ for $\eta$ Car's distance of $2.6 \mathrm{kpc}$.

Then, we analysed MECS and PDS simultaneously, allowing a PDS/MECS normalisation factor of 0.85 , to take into account the miscalibration between the two instruments. This correcting factor was also used in Paper I, although it was not explicitly indicated. As shown in Fig. 3, PDS counts are well above the extrapolation to higher energies of the MECS best fit thermal model. The difference between observed and expected flux increases with energy, suggesting the presence of an additional very high energy component.

To account for the high energy tail, we tried to fit the 2-150 keV spectrum with two thermal components. However, the fit of MECS+PDS with two freely varying temperatures always gives unrealistic results. Therefore, we fit MECS+PDS with frozen $k T(1)=5.51 \mathrm{keV}$ and $\mathrm{NH}(1)=4.82 \times 10^{22} \mathrm{~cm}^{-2}$, and constrain $k T(2)$ to be close to $10 \mathrm{keV}$. We thus derived: $k T(2)=14 \pm 5 \mathrm{keV}$, and $\mathrm{NH}(2)=(543 \pm 22) \times 10^{22} \mathrm{~cm}^{-2}$, with a reduced $\chi^{2}$ of 2.33 (Fig. 4). However, this result is not convincing, not only because the fit runs below the observed flux above $30 \mathrm{keV}$, but also because an unlikely very high $\mathrm{NH}$ is required for the higher temperature component. This suggests that the high energy spectrum of $\eta$ Car could be non-thermal.

Therefore, we tried to reproduce the $2-150 \mathrm{keV}$ spectrum with a combination of thermal bremsstrahlung (with frozen $k T=5.70 \mathrm{keV}$ ) and power law components, having the same 


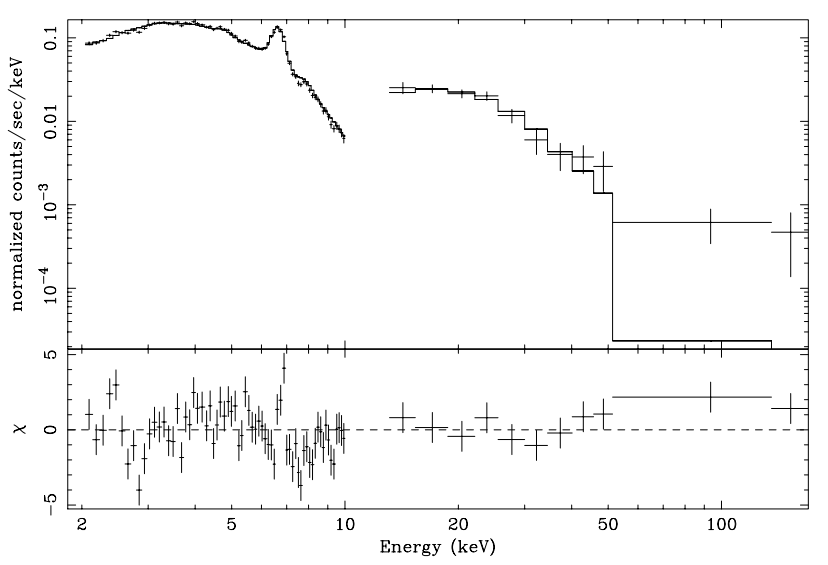

Fig. 4. Upper panel: results of the fit of the BeppoSAX MECS and PDS (2-150 keV) spectrum of $\eta$ Car in June 2000 with a two-temperature (5.5 keV and $14 \mathrm{keV}$ ) MEKAL model. The residuals are plotted in the lower panel.

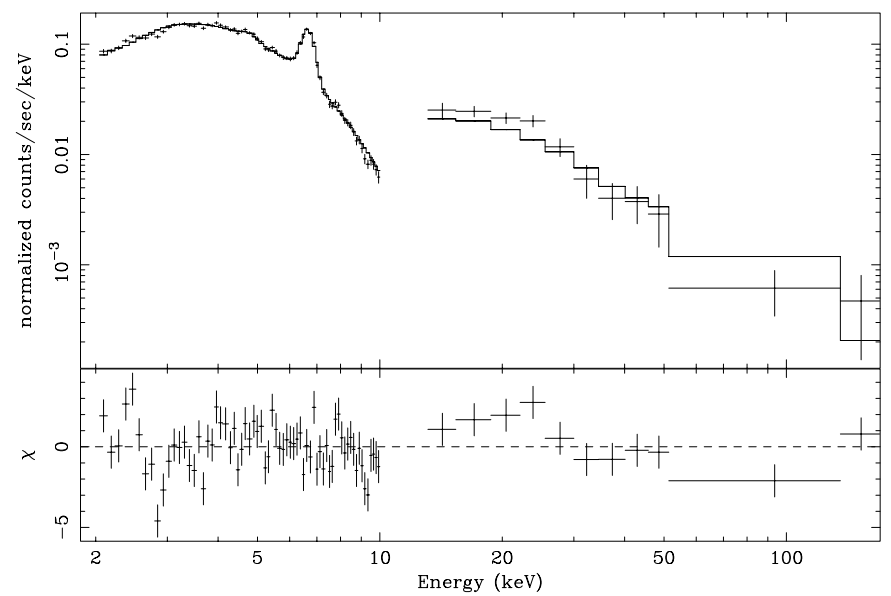

Fig. 5. The BeppoSAX MECS and PDS (2-150 keV) spectrum of $\eta$ Car fitted with a $5.70 \mathrm{keV}$ thermal bremsstrahlung plus a power law spectrum with photon index 2.42, and a Gaussian line centred at $6.70 \mathrm{keV}$.

absorption column density $\left(4.82 \times 10^{22} \mathrm{~cm}^{-2}\right)$, and a Gaussian line at $6.7 \mathrm{keV}$. The best fit model is shown in Fig. 5. The nonthermal component has a best fit photon index of $2.42 \pm .04$ (reduced $\chi^{2}=2.41$, for 79 d.o.f.). If produced in a shock, this photon index would imply a compression ratio for the shock front of $\chi=3.1$, thus the shock should be relatively weak (White \& Chen 1994). The nonthermal component in the 13-150 keV range has an integrated energy flux of $5.51 \times$ $10^{-11} \mathrm{erg} \mathrm{cm}^{-2} \mathrm{~s}^{-1}\left(11.6 L_{\odot}\right)$, a value comparable to that of the thermal $5 \mathrm{keV}$ component.

We have also attempted to fit the overall MECS+PDS spectrum with a single absorbed power law spectrum plus a Gaussian line. Unexpectedly, quite a good fit was obtained (excluding the photon events below $2 \mathrm{keV}$ ) with a best fit photon index of $2.467 \pm 0.024, \mathrm{NH}=(6.46 \pm 0.12) \times 10^{22}$, an $\mathrm{Fe}$ line centred at $6.733 \pm 0.007 \mathrm{keV}$, and a reduced $\chi^{2}$ of 2.404. However, this solution appears unrealistic, in particular because it would imply that the iron emission is mostly the $6.4 \mathrm{keV}$ fluorescent line, in disagreement with the measured peak energy of the feature $\left(E_{\text {cor }}=6.70 \pm 0.01 \mathrm{keV}\right)$.
Therefore, the thermal plus power law model appears more likely to us. Incidentally, we notice that the two power law spectra have nearly the same slope, independent of the energy range considered.

\section{Discussion}

The high energy X-ray spectrum of $\eta$ Car is challenging, both because such an energetic and powerful source has so far never been seen in any other star-like source and no current model for non-thermal emission seems applicable to the case of $\eta$ Car.

In the $\eta$ Car binary model it is assumed that the thermal ( $5 \mathrm{keV}$ ) X-ray emission originates in the hot shocked gas produced by collision between the winds of the two stellar components (e.g. Pittard et al. 1998; Ishibashi et al. 1999). The shocked region should be placed at a distance from a few to many $\mathrm{AU}$ from the surface of the $\mathrm{S}$ Dor star during the highly eccentric orbital motion of the system. In this model, the plasma temperature is linked to the wind velocities, and would be unable to produce directly the highest energy photons seen in $\eta$ Car.

The power law spectrum exhibited by $\eta$ Car above $10 \mathrm{keV}$ indicates the presence of an additional non-thermal source. In principle, non-thermal emission can be explained by several physical processes, most of which are, however, barely compatible with current models of $\eta$ Car. A plausible, but still difficult to accept process would be inverse Compton scattering (IC) of low frequency photons by high energy electrons. In the case of $\eta$ Car a powerful photon source could be represented by the intense ultraviolet radiation from the stellar components of the binary system, probably from the hot secondary, since most likely the UV photons from the S Dor primary do not emerge from its dense wind. Stellar UV photons scattered by relativistic electrons would finally emerge with energies in the $\mathrm{X}$ - and $\gamma$-ray range, and may carry away much of the energy pumped into the electrons at shocks.

This process would require the presence of relativistic electrons with $\gamma \sim 100$. According to White (1985) and Chen \& White (1991) in the winds of early-type stars electrons can be accelerated to relativistic energies via the first-order Fermi mechanism in strong isothermal shocks generated by radiation-driven instabilities in the wind. On the other hand, Jardine et al. (1996) have shown that in a colliding wind binary system, electrons can be accelerated up to relativistic energies in a current sheet, formed when the magnetic fields carried in the stellar winds are forced together as the winds collide. In the case of $\eta$ Car there is no direct or indirect evidence of the presence of very high energy electrons.

Indirect evidence could be non-thermal radio emission, like that detected in galactic and extragalactic high energy $\mathrm{X}$-ray sources. However, non-thermal radio emission centred on $\eta$ Car's stellar core has not been detected although we cannot exclude its presence. Were it present, it would lie below the surface that is optically thick due to free-free emission, and the whole central region of $\eta$ Car is optically thick in radio wavelengths to out several arcseconds. Since the non-thermal emission should not extend that far away from the shock, it should be completely hidden by the thermal emission from the 
dense ionised stellar winds (Stephen White, priv. comm.). One should also consider that, as recently discussed by Dougherty et al. (2003), in colliding wind binary systems the non-thermal radio emission could be weakened by self-absorption and inverse Compton cooling.

Non-thermal radio emission has been detected in a number of massive binaries, suggesting the presence of relativistic electrons with power-law spectra, which could result in hard X- and $\gamma$-ray non-thermal flux via IC scattering (e.g. Benaglia \& Romero 2003). Among them, the WR+O binary system WR 140 bears some resemblance to the $\eta$ Car system for its long period (7.9 yr), and high orbital eccentricity (e.g. Marchenko et al. 2003), and for the presence of a variable IR excess attributed to dust formation near periastron (e.g. Williams et al. 1987). The X-ray emission of WR 140 is one order of magnitude smaller than that of $\eta$ Car, but, like $\eta$ Car, it suffers a strong absorption excess near phases 0.03 and 0.08 (Zhekov \& Skinner 2000). If the physical process of production of non-thermal radio emission (that is of high energy electrons) in WR 140 is also working in $\eta$ Car, we would expect from $\eta$ Car a very strong non-thermal radio emission. As discussed above, its absence is probably due to the heavy obscuration by the thermal absorption from the dense stellar winds. Indeed, the crucial point of the spectrum $\eta$ Car's core radio emission remains unsolved.

Self-Comptonisation is a mechanism proposed to explain non-thermal emission in AGNs, as well as in galactic compact X-ray sources. As for instance discussed by Hua \& Titarchuk (1995), quasi-power law X-ray spectra can be produced by diffusion of low frequency photons by optically thick relativistic plasma clouds. In this framework, we might assume that the spectrum of $\eta$ Car above $10 \mathrm{keV}$ is produced by scattering of the $5 \mathrm{keV}$ thermal radiation itself by high energy $(>10 \mathrm{keV})$ electrons present in the shocked region. This seems to us a more promising process as it does not require highly relativistic electrons as in the IC case.

The study of the spectral variability along the $5.53 \mathrm{yr}$ cycle could be a complementary way to tackle the problem of the origin of the high energy X-ray tail of $\eta$ Car. Table 1 reports the PDS count rates and the best fit parameters of the thermal component during the four BeppoSAX observations. We recall that, according to the RossiXTE observations, in December 1997 $\eta$ Car underwent a deep X-ray eclipse, which is currently associated with the periastron passage of the suggested binary system (Ishibashi et al. 1999). The X-ray eclipse lasted until the end of February 1998. Hence, the BeppoSAX observation of mid March 1998 was made during $\eta$ Car's egress from the eclipse.

Figure 6 compares the BeppoSAX MECS spectra observed during the four epochs. For the sake of homogeneity with the other observations, only MECS units 2 and 3 have been considered for the December 1996 spectrum. It is evident in the figure that beyond the iron feature the four spectra nearly overlap, also suggesting that the highly absorbed March 1998 spectrum had about the same temperature and unabsorbed flux as in the other three epochs.

In three cases the PDS count rate in the $13-20 \mathrm{keV}$ range was the same within the data uncertainty. As shown in Table 1,

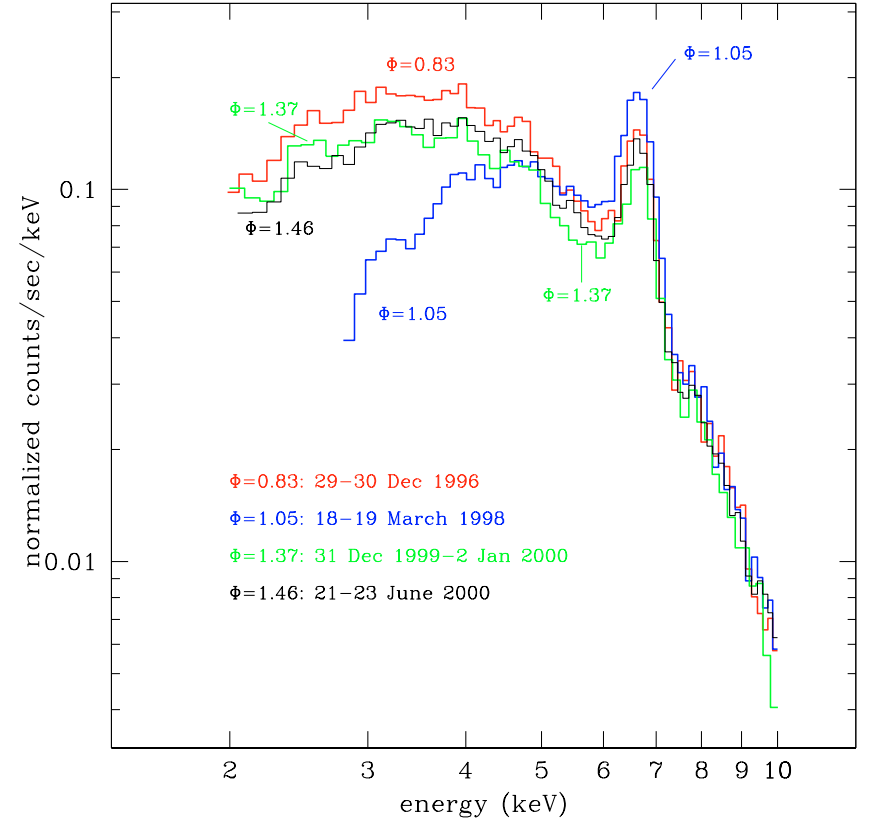

Fig. 6. Plot of the BeppoSAX MECS23 spectra of $\eta$ Car during December 1996-June 2000. The phases of the $5.53 \mathrm{yr}$ cycle are indicated. Note the larger flux of the iron line in March $1998(\Phi=1.05)$ when the spectrum was strongly absorbed. At energies $>7 \mathrm{keV}$ the four spectra overlap.

in March $1998 \eta$ Car was not detected with PDS. The $3 \sigma$ upper limit of $0.15 \mathrm{~s}^{-1}$ is equal to the flux measured in the other three observations, but we think that this coincidence is accidental, and that in March 1998 the high energy tail was weak. Most probably, this component followed the trend of the thermal one, and greatly weakened during the 1998.0 X-ray eclipse. Our March 1998 observation suggests that, while the thermal component had already recovered its luminosity, although with a large residual absorption, the high energy tail was still weak. It should have recovered its luminosity at a later time (but well before phase 1.37). It may be noticed that a long recovering time was also found in the visual spectrum for the high excitation emission lines (e.g. [Ne III]), which, as discussed in Paper I, were still very weak in May 1998. The limited time-coverage does not allow us to conclude whether there is a physical link between the two phenomena, but it would be worthwhile investigating this in the future.

\section{Conclusion}

We have presented the first in-depth analysis of the spectrum of the $\eta$ Car system above $10 \mathrm{keV}$, based on a long exposure BeppoSAX observation. The power law best fit suggests a non-thermal origin of the hard X-ray tail. The integrated $13-150 \mathrm{keV}$ luminosity $\left(\sim 12 L_{\odot}\right)$ is comparable to the luminosity of the $2-10 \mathrm{keV}$ thermal component $\left(19 L_{\odot}\right)$, and suggests the presence of a very effective formation process.

Two models have been considered in the light of the proposed binary nature of $\eta$ Car. In one model the high energy tail is produced by inverse Compton scattering of the UV stellar photons by relativistic electrons produced in the 
wind of the primary star, or in the shocked colliding wind region. Alternatively, the high energy photons are produced by self-Comptonisation of the thermal $5 \mathrm{keV}$ emission from relativistic electrons with energies much lower than in the previous case. However, the weakness of the high energy tail in March 1998 could be better explained as high inverse Compton cooling of the relativistic electrons during periastron passage, when the colliding wind shock is closer to the stars. Neither model has enough support from observations, such as the detection of non-thermal radio emission from the central source, a point which would require new very high resolution radio observations.

A crucial point for understanding the nature of the high energy tail of $\eta$ Car is to investigate the slope of the spectrum at higher energies and to measure the high energy cutoff which is related to the energy of the scattering particles. Our PDS observations allow us to determine a lower limit to the cutoff energy of $\sim 50 \mathrm{keV}$. INTEGRAL observations might allow measurement of the X-ray spectrum of $\eta$ Car above $100 \mathrm{keV}$, and to determine up to what energy the power law spectrum extends.

Finally, new high energy ( $>10 \mathrm{keV})$ X-ray observations, e.g. with the proposed ASTRO-E satellite, of $\eta$ Car near the periastron passage of the system will provide a clue to where the non-thermal source is located. Our March 1998 upper limit might suggest a recovery time slower than that of the thermal source, as observed in the optical spectra in the high energy emission lines. It would important to investigate the physical link beteen the two phenomena, and whether that behaviour is associated with the high $\mathrm{NH}_{h}$ value found also at the eclipse egress.

Acknowledgements. We are grateful to the other BeppoSAX team members at ASDC for help in acquiring and reducing the data. Thanks are due to L. Piro, V. F. Polcaro and Stephen White for discussions, and to the anonymous referee for constructive suggestions. This work is partly based on contract $\mathrm{I} / \mathrm{R} / 053 / 02$ of the Italian Space Agency (ASI).

\section{References}

Andriesse, C. D., Donn, B. D., \& Viotti, R. 1978, MNRAS, 185, 771 Benaglia, P., \& Romero, G. E. 2003, A\&A, 399, 1121
Boella, G., Chiappetti, L., Conti, G., et al. 1997, A\&AS, 122, 327

Chen, W., \& White, R. L. 1991, ApJ, 381, L63

Chen, W., \& White, R. L. 1994, Ap\&SS, 221, 259

Corcoran, M. F., Ishibashi, K., Swank, J. H., \& Petre, R. 2001, ApJ, 547,1034

Damineli, A., Kaufer, A., Stahl, O., Lopes, D. F., \& de Araujo, F. X. 2000, ApJ, 528, L101

Dougherty, S. M., Pittard, J. M., Kasian, L., et al. 2003, A\&A, 409, 217

Duncan, R. A., \& White, S. M. 2003, MNRAS, 338, 425

Frontera, F., Costa, E., Dal Fiume, D., et al. 1997, A\&AS, 122, 357

Hillier, D. J., Davidson, K., Ishibashi, K., \& Gull, T. 2001, ApJ, 553, 837

Hua, X.-M., \& Titarchuk, L. 1995, ApJ, 449, 188

Ishibashi, K., Corcoran, M. F., Davidson, K., et al. 1999, ApJ, 524, 983

Jardine, M., Allen, H. R., \& Pollock, A. M. T. 1996, A\&A, 314, 594

Manzo, G., Giarrusso, S., Santangelo, A., et al. 1997, A\&AS, 122, 341

Marchenko, S. V., Moffat, A. F. J., Ballereau, D., et al. 2003, ApJ, 596, 1295

Oosterbroek, T., Parmar, A. N., Mereghetti, S., \& Israel, G. L. 1998, A\&A, 334, 925

Parmar, A., Martin, D. D. E., Bavdaz, M., et al. 1997, A\&AS, 122, 309

Pittard, J. M., Stevens, I. R., Corcoran, M. F., \& Ishibashi, K. 1998, MNRAS, 299, L5

Pittard, J. M., \& Corcoran, M. F. 2002, A\&A, 383, 636

Rebecchi, S., Viotti, R. F., Grandi, P., et al. 2001, in X-Ray Astronomy 2000, ed. R. Giacconi, S. Serio, \& L. Stella, ASP Conf. Ser., 234, 107

Tiengo, A., Göhler, E., Staubert, R., \& Mereghetti, S. 2002, A\&A, 383, 182

van Boekel, R., Kervella, P., Schöller, M., et al. 2003, A\&A, 410, L37

Viotti, R. 1995, Rev. Mex. Astr. Ap. Conf. Ser., 2, 1

Viotti, R., Corcoran, M. F., Damineli, A., \& Grandi, P. 1998, Proc. Symp. The Active X-Ray Sky, ed. L. Scarsi et al., Nuclear Phys. B (Proc. Suppl.), 69/1-3, 36

Viotti, R. F., Antonelli, L. A., Corcoran, M. F., et al. 2002a, A\&A, 385, 874 (Paper I)

Viotti, R. F., Antonelli, L. A., Rebecchi, S., \& Rossi, C. 2002b, JApA, 23, 19

White, R. L. 1985, ApJ, 289, 698

White, R. L., \& Chen, W. 1994, Ap\&SS, 221, 295

Williams, P. M., van der Hicht, K. A., van der Woerd, H., et al. 1987, in Instibilities in Luminous Early type stars, ed. H. J. G. Lamers, \& C. W. H. de Loore (Dordrecht: Reidel), 221

Zhekov, S. A., \& Skinner, S. L. 2000, ApJ, 538, 808 